\title{
The Study of Crew Physical and Psychological Evaluation and Sports Intervention Expert System
}

\author{
Guoqing $\mathrm{Li}^{\mathrm{a}}$ Bin Zhang ${ }^{\mathrm{b}}$ \\ Department of physical and training, Harbin Engineering University, China, 150001 \\ aliguoqing@hrbeu.edu.cn, b'zhangbin2214@hrbeu.edu.cn
}

Keywords: Expert System, Physical and Psychological Evaluation, Sports Intervention

\begin{abstract}
The crew health evaluation model based on probability neural network and the sports items intervention expert system established in the paper. The system can track and guide $n$ the crew physical and psychological health status at sea in real time. The research work has important practical significance and great practical value, which may enhance the crew physical and psychological health, improve work efficiency, promote the harmony and stability of the ship small environment, maintain the normal life order and ensure safety at sea.
\end{abstract}

\section{Introduction}

Ocean shipping has the characteristics of drifting time longer at sea, away from land and shore. So the crew's physiological status, psychological status and level of technology have put forward higher requirements. It has been shown that human misplay is the main cause of ship accident factors from the large number of maritime case analysis at internal and abroad. Therefore, it is of great significance to analyze and research the physical and psychological status. Which can take the corresponding methods and preventive measures to reduce the dynamic human misplay factors, as we known these factors would cause ship mechanical accident. This paper will made crew physical and psychological health evaluation model for ocean shipping at sea eventually, to track and evaluate crew health status, take intervention measures, reduce human accidents. System frame as shown in figure 1 . The research field of this paper is in the red line range.

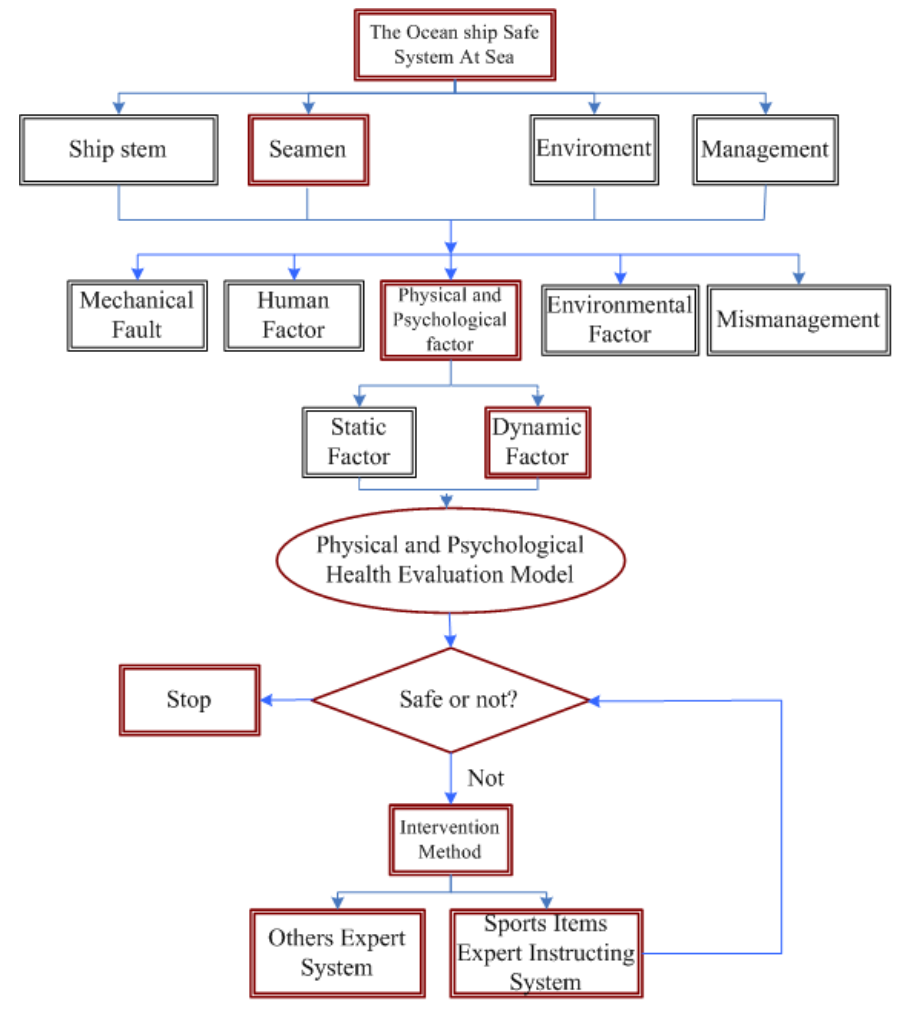

Fig.1 Block diagram range of study 


\section{Theory of Probability Neural Network}

Probability neural network (PNN) was introduced by Specht in1988 based on Parzen probabilistic density function and Bayesian classification rule (Specht, 1990)[1] . It can be used for direct estimation of posterior probability densities and pattern classification. Competition neural cell accept the distance vector and computes the probability of each model. If the probability is the most, the output is 1 . Otherwise, the output is 0 . In the process of training, the training samples are stored in network. The Gauss function is assessed by statistic of experience[2].

\section{Crew Physical and Psychological Health Evaluation Model}

Definition: the research is limited in the dynamic factors of the ocean crew physical and psychological health evaluation index, variable factors, adjustable factors, controllable factors)

Definition \Criterion of Physical and Psychological Health. Definition of health (WHO definite): Physical, psychological and social adaptation is in good condition. Six criterion of the psychological health (from WHO).

(1) Who has a good sense of self, can do something consciously. Who can be gratified, self-esteem, self-confidence facing excellence and good qualities of their own and not be depressed even give up because of their own shortcomings.

(2) Who is calm facing reality, has the higher reality ideal, also can correctly deal with the defect and the setback, achieve, that means "win without tender, defeated without discouragement ".

(3)Who maintain normal relationship, admit others, limit yourself. In getting along with others, respect is more than jealous, trust than suspected, love than hate.

(4)Who has strong emotion control ability, can maintain emotional stability and psychological balance. Their response is moderate facing the outside stimulus and their behavior is coordination.

(5) Who do things optimistically, full of hope, always maintain a positive and enterprising attitude.

(6) Who cherish life, love life, and have the philosophy of life consistently.

Establishment the configuration of PNN. The ocean crew physical and psychological health at sea evaluation model chose the index of fatigue, sleep, appetite, food egestion, loneliness, anxiety, depression, panic, emotionality tension, thinking ability, self-control ability as the input of PNN, and crew health level, health, sub-health, unhealthy, serious unhealthy as output. Some of the variables results of the physical and psychological health were chosen as PNN training set. We can slide the block to show state of crew health to achieve the evaluation result. Please see fig.2.

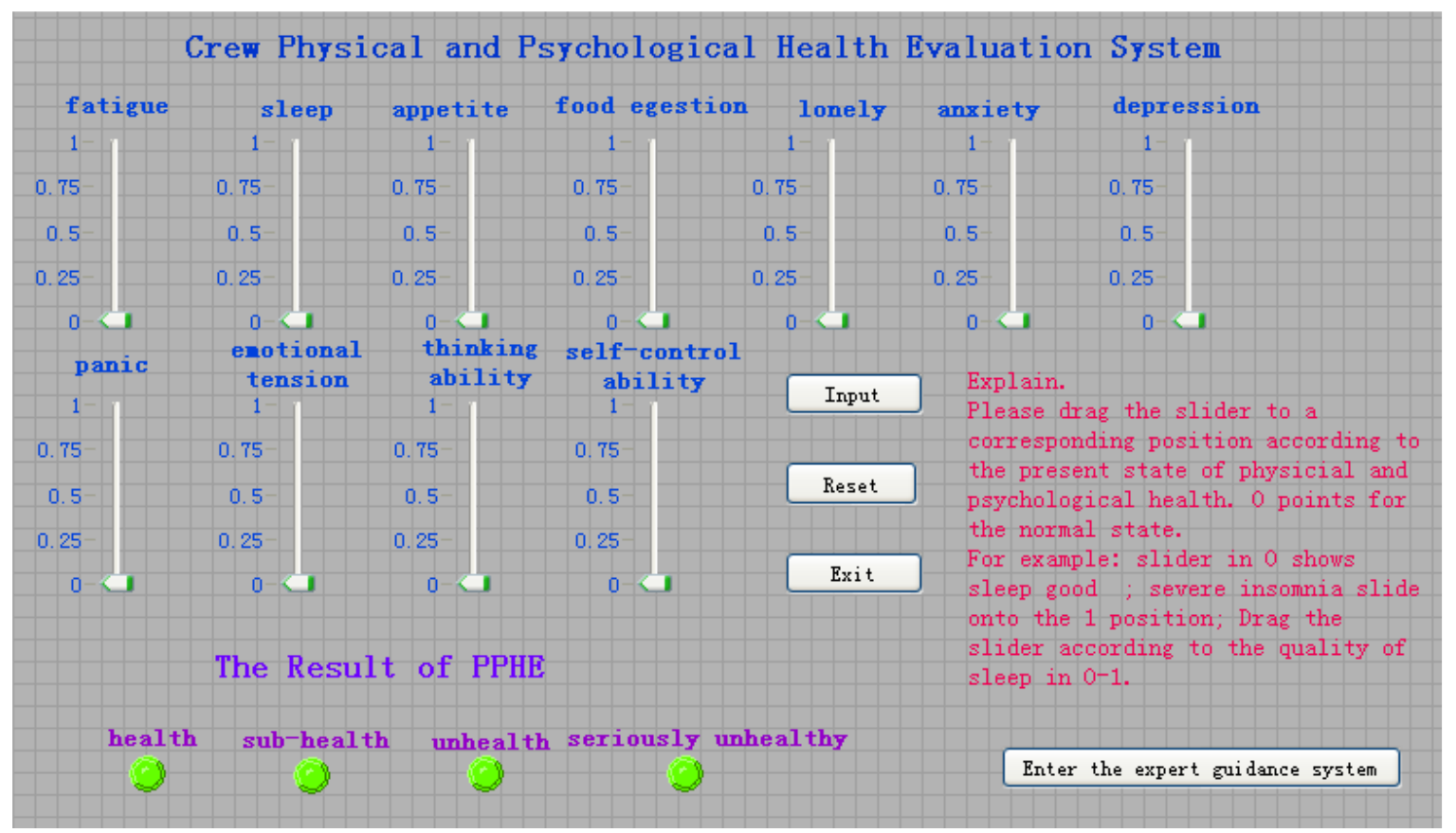

Fig.2 Interface of health evaluation system base on PNN 
Since centralized degree of the sample data is high, the data is processed by normalization. Every feature is in the $0-1$ interval. The interval value indicated the degree, for example: if the sleep state is good, then the value is recorded 0.00 , insomnia recorded 1.00. The state of sleep quality drop a little, but can be a good rest, recovery is perfect, recorded 0.60 . Sleep quality drops badly, the recovery is bad, recorded 0.40. MATLAB software is used to train sample set.

\section{The sports items intervention expert system}

Sports project selection principle of crew health state intervention. According to the physical and psychological health state as mentioned above, this paper put forward the exercise treatment, chose several easy to carry out on board. These sports have better effect on common physical and psychological illness. Brief introduction is as followed[3-6].

Tai Chi. Body joints, muscles and bones will each pull, press and be diastolic, when playing Tai Chi. Massage effect will be in Visceral due to abdominal breathing, and it is beneficial for intestinal peristalsis with increasing of diaphragmatic upper and lower amplitude. In addition, Tai Chi exercise has obvious effect to reduce hypertension. Some studies show that blood pressure may drop 10 $20 \mathrm{mmHg}$ after a set of Tai Chi. As the long sea voyage process is long, the crew has lots of spare time and are prone to anxiety and irritability. It is beneficial for them to practice Tai Chi at sunrise or sunset on the spacious deck in the vast sea. Tai Chi can train the crew to be happy and pleased with themselves, and be in a calm mood.

Ear action practice. The helix covers the ears when doing exercise, rub the earhole with the root of palm (i.e. massage earhole that be covered by helix), Rub to the ear feel itch, when the blood circulate to the ear. It is help to relief tinnitus.

Sit-ups. This sports can exercise the leg strength also has very good effect on the waist, of course you can use the squat method.

Barbell practice. Carry barbell with shoulder, helps to improve renal function, also can achieve the effect of alleviating tinnitus.

Walking on the deck. Walking exercise method is very simple, because the tonnage of ocean shipping is very large. The broad deck area is equivalent to two football fields. The crew can make full use of the convenient conditions, when the rising in every morning the crew can enjoy the beauty on the sea and walk or jog on the open deck. This sport not only may increase the cardiopulmonary function, but also can cultivate their original nature, condition psychology. Slow walking is $60-80$ steps per minute, middle speed is 80-100 steps per minute, fast 100-120 steps. In order to increase effect, auxiliary action of the upper limbs expanding chest can do simultaneously. At present in China the method of 12 minutes walking is adopt. That means try your best to walk as far as long distance parallel in 12 minutes, persist in everyday. This sports mode has good effect on rehabilitation and prevention of bronchitis for crew.

Climbing gangway ladder. A certain number of gangways are in ship limited area. The crew can enhance their ability of respiratory system by climbing the gangway ladder coordinating with the movement of breath training. First suction with the nose, then narrow lips to breathe out, each climb2 steps breathe again, this sport can be realized on every ship.

Comfortable and slow motion in cabin. The crew work on board for long-term, often climb cabin, so is very tired. In e course of time they will be facing lumbago and skelalgia, which can not relieve by energetic exercise, but massage, and do moderate exercise. For deck narrow, poor conditions of the ship, the crew can take Comfortable and slow motion in cabin to for rehabilitation. The concrete forms can reference interface of expert system. Please see fig. 3

The interface of crew health evaluation model and sports items intervention expert system Please see the interface of sports intervention expert system. In expert system we made a data base to save the sports expert experiments. This interface have given the sub-health advises of sports expert. 


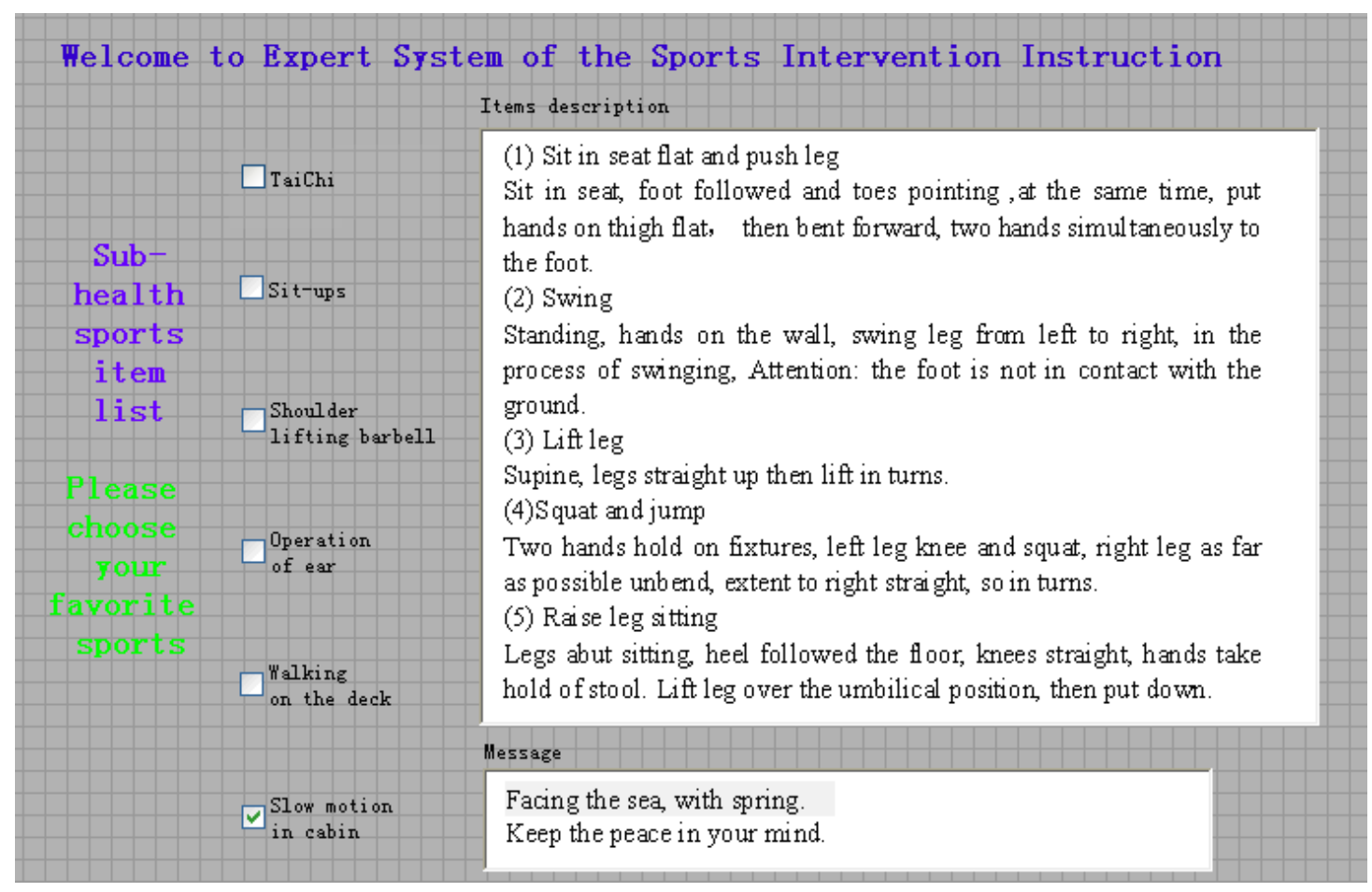

Fig.3 Sport intervention expert system

\section{Conclusion}

The artificial neural network theory is applied to the crew health evaluation, according to the crew health evaluation index. The corresponding PNN for health state evaluation has been established. Training samples of neural network obtained using the expert experience and statistical data. The network was trained while the accuracy met requirements. With the aid of computer and MATLAB software, the evaluation results can be conveniently achieved. PNN model that was trained can be operated "off-line", evaluate the crew physical and Psychological health status accurately and effectively. And the guidance and recommendations of sports items intervention measures were given by crew health expert system.

\section{References}

[1] D.Specht. Introduction to the Modified Probabilistic, Neural Networks.3 (1990)109-118.

[2] XING Chuan-ding, YANG Jia-ming, REN Qing-sheng, Theory of artificial intelligent and its application. ShangHai, DongHua university press,2005

[3] A.P. Eremeev, V.N. Vagin, A real-time decision support system prototype for management of a power block, International Journal Information Theories \& Applications. 10(2005)248-255.

[4] Chen Meiqin, Qiao Feiyue, Research on Sports Effect of Occupation Disease Prevention and Treatment for Crew, Science and Technology Innovation Herald. 35(2011)5,16 .

[5] Gongyi, Discuss Effect of Physical Exercise on The Positive role of Sub Health, Journal of Yichun University, 32(2010)12 